\title{
Phase Transitions of Soluble Surfactants at a Liquid-Vapor Interface
}

\author{
M. S. Tomassone ${ }^{1}$, A. Couzis ${ }^{2}$, C. Maldarelli ${ }^{1,2}$, J. R. Banavar ${ }^{4}$ and J. Koplik ${ }^{1,3}$. \\ Benjamin Levich Institute ${ }^{1}$ and Departments of Chemical Engineering ${ }^{2}$ and Physics ${ }^{3}$ \\ City College of the City University of New York, New York, New York, 10031 \\ ${ }^{4}$ Department of Physics and Center for Materials Physics \\ The Pennsylvania State University, University Park, PA 16802
}

(October 29, 2018)

\begin{abstract}
Although medium chain length insoluble amphiphiles are well known to form gaseous and liquid expanded phases on an air/water interface, the situation for the soluble case is less clear. We perform molecular dynamics simulations of model surfactant molecules dissolved in a bulk liquid solvent in coexistence with its vapor. Our results indicate a transition in both soluble and insoluble surfactants: a plateau in surface tension vs. surface coverage, whose instantaneous configurations display two phase coexistence, along with correlation functions indicating a transition to gaseous to liquid-like behavior.
\end{abstract}

PACS numbers: 79.20.Rf, 64.60.Ht, 68.35.Rh

Surfactants are amphiphilic molecules which contain a polar head group and a nonpolar tail, which usually consists of a chain of hydrocarbon groups. At an air/water interface surfactants arrange themselves with their polar group immersed in water, interacting by dipolar forces, while the hydrocarbon tails are displaced outward into the air. The presence of surfactants at the air/water interface lowers the free energy, and in turn the surface tension, usually as a non-increasing function of the concentration of surfactant in the liquid [1]. The degree of aggregation and molecular ordering of surfactants adsorbed at an air/solvent interface is reflected in the appearance of distinct surface phases as a function of surfactant concentration. In addition to the obvious two dimensional Gas $(\mathrm{G})$ and disordered liquid phases (more precisely, the Liquid Expanded LE phase), it is possible to find condensed mesophases and semi-solid crystalline-like phases in monolayers [2,3].

This paper focuses on the determination of soluble surfactant adsorption isotherms simultaneously with their phase behavior and surface tension variation, and in particular the G/LE transition, using Molecular Dynamics (MD) simulations. In the context of insoluble surfactants, this approach has been used extensively to study the structure of monolayers restricted to the surface of a substrate 4 6], obtaining information both complementary to and in semi-quantitative agreement with experiment on Langmuir films. For soluble surfactants there is emerging experimental evidence for the existence of gaseous, liquid, and condensed surface phase transitions [7], but no accompanying theory or simulation. There are MD simulations of soluble surfactant monolayers [8], but these do not study the surface-bulk equilibrium which gives rise to the surface phases, or the transitions between them. In contrast, MD simulations in liquid-liquid systems [9] have studied the equilibrium between the liquid phase and monomeric and surfactant aggregates in the bulk, but not surface phase transitions.
Our simulations model surfactant molecules placed in a solvent in equilibrium with its vapor, which then migrate to the surface to a degree controlled by the choice of interaction potential. We observe that as their initial concentration increases, the surfactant structure on the liquid/vapor interface ranges from a gas phase containing isolated molecules or small clusters, to a coexisting mixture of clusters of various sizes, to a single disordered spanning liquid cluster. Examination of positional and tail-orientation distribution functions show a gradual transition to liquid-like ordering, and the isotherm of surface tension $v s$. surface concentration exhibits a plateau indicative of a phase transition. We have studied both insoluble and soluble surfactant systems, obtaining rather similar behavior, but in this Letter we concentrate on the latter. Further details will be presented elsewhere [10].

Our calculations are based on standard MD techniques using Lennard-Jones (LJ) interactions, in an NVT ensemble [11]. The potential between any two atoms of type $i$ and $j$ separated by distance $r$ is $V_{i j}^{L J}(r)=$ $4 \varepsilon\left[(\sigma / r)^{12}-C_{i j}(\sigma / r)^{6}\right]$. Here $\epsilon$ is an energy scale, $\sigma$ is approximately an atomic diameter, and the characteristic microscopic time unit is $\tau=\sqrt{\left(m \sigma^{2} / \varepsilon\right)}$, with atomic mass $m$. The adjustable coefficients $C_{i j}$ determine the interactions between the various molecular species and control the chemistry of the system. The surfactant atoms are bound in chain molecules using the Finitely Extensible Nonlinear Elastic (FENE) potential 12] $V^{F E N E}=-\frac{1}{2} K r_{0}^{2} \ln \left[1-\left(r / r_{0}\right)^{2}\right]$ which acts only between adjoining atoms on a chain; $r_{0}$ is the maximum bond length and $K$ is a spring constant. The system is maintained at a temperature $T=0.9 \epsilon / k$ by a constant kinetic energy thermostat. We have worked with a system of 11520 atoms in total, with surfactant chains of length 6 . The atoms move in a three dimensional box of size $20.5 \sigma \times 20.5 \sigma \times 68.4 \sigma$, with periodic boundary conditions in all directions, and form a horizontal liquid 
slab with vapor above and below, with two (statistically) planar liquid/vapor interfaces. A snapshot of a typical configuration is shown in Fig. 17a.

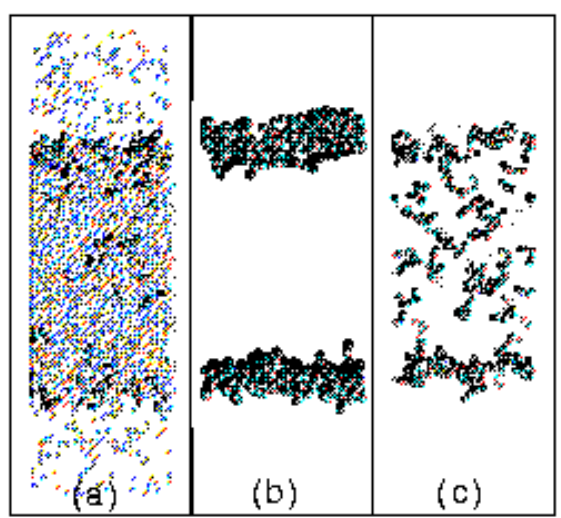

FIG. 1. Snapshots of the simulated system; (a) all atoms in a soluble case, (b) surfactant molecules only in an insoluble case, (c) surfactant molecules only in a soluble case.

The surfactant molecules are initially distributed at random in the liquid, and then migrate to the liquidvapor interface to a degree determined by the choice of $C_{i j}$, so that the solubility is indirectly under control. An insoluble surfactant is achieved by choosing a strong attraction between head group and solvent, an antipathy between surfactant tail and solvent, and a strong cohesion among the molecules in the solvent. The full set of interaction coefficients is shown in Table I, where the subscript convention is $\mathbf{1}$ : hydrophilic head, 2: hydrophobic tail and 3: solvent. A typical snapshot of the insoluble surfactant phase alone is shown in Fig. 1 b. For a soluble surfactant system, we modify the interactions to allow surfactant to remain in solution. A first ingredient is to reduce the antipathy between the surfactant tails and the solvent, by increasing $C_{23}$, and the second is to reduce the cohesion of the solvent, by reducing $C_{33}$, and the full set is shown in Table II,

The surface tension is computed from the standard microscopic expression for a planar interface [13], $\gamma=$ $\frac{1}{A}\left\langle\sum_{i<j} \frac{r_{i j}^{2}-3 z_{i j}^{2}}{2 r_{i j}} \frac{d V\left(r_{i j}\right)}{d r_{i j}}\right\rangle$, where the sum runs over all interacting pairs of atoms, $V$ is the full interatomic potential, $A$ is area of the interface, and the angle brackets denote a time average. The insoluble surface tension isotherm is given in Fig. 2. There is a clear reduction for small amounts of surfactant, followed by an approximate plateau until a critical concentration is reached, and then a rapid decrease. (At still higher surfactant concentration we observe buckling of the interface or micelle formation.) The structure of the surfactant layer may be inferred from the pair distribution function $g(r)$ [14], here taken to be the probability that two head groups on a given interface are separated

Table I: Insoluble.

\begin{tabular}{||r|rrr||}
\hline $\mathrm{C}(\mathrm{i}, \mathrm{j})$ & $\mathbf{1}$ & $\mathbf{2}$ & $\mathbf{3}$ \\
\hline $\mathbf{1}$ & 1.0 & 1.0 & 3.0 \\
$\mathbf{2}$ & 1.0 & 0.2 & 0.6 \\
$\mathbf{3}$ & 3.0 & 0.6 & 1.15 \\
\hline
\end{tabular}

Table II: Soluble.

\begin{tabular}{||r|rrr||}
\hline C(ij) & $\mathbf{1}$ & $\mathbf{2}$ & $\mathbf{3}$ \\
\hline $\mathbf{1}$ & 1.0 & 1.0 & 3.0 \\
$\mathbf{2}$ & 1.0 & 0.2 & 0.8 \\
$\mathbf{3}$ & 3.0 & 0.8 & 1.10 \\
\hline
\end{tabular}

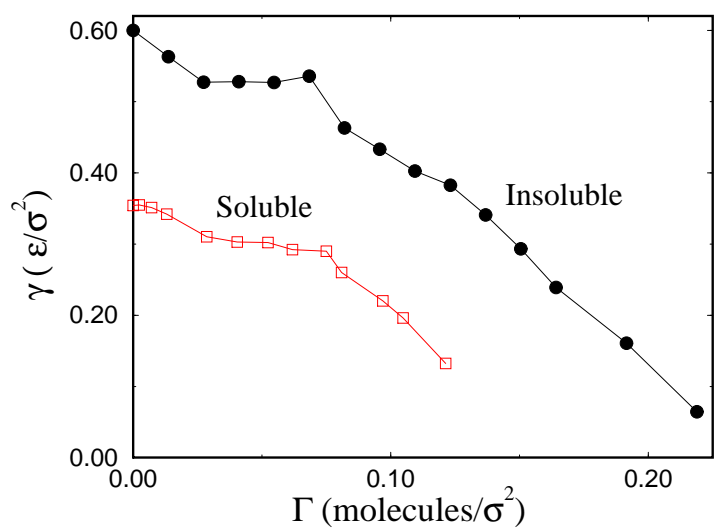

FIG. 2. $\gamma-\Gamma$ isotherms for the insoluble and soluble cases.

by a distance $r$. As we see in Fig. 3a, in the very low coverage region $g(r)$ is a flat line typical of a gas, but as the coverage increases up to the end of the plateau, the curve changes to that of a typical liquid, with a prominent nearest-neighbor first peak, a rather broad second peak exemplifying short range order, and some further structure at larger $r$ as well. This simulation therefore reproduces the well-documented G/LE phase transition for insoluble surfactant.

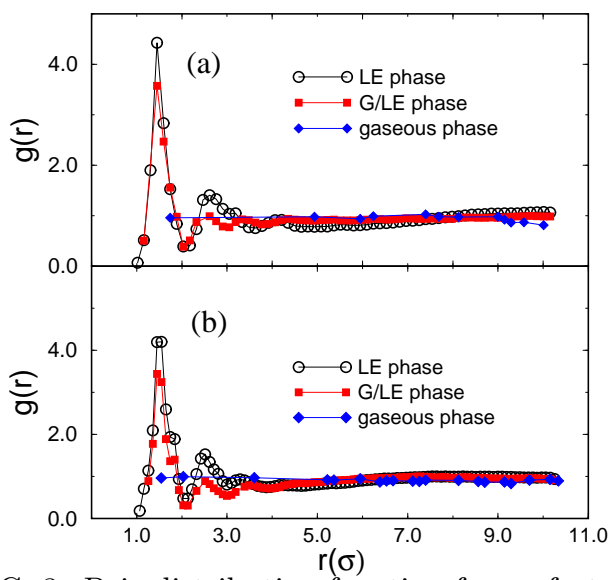

FIG. 3. Pair distribution function for surfactant monolayers on the liquid-vapor interface: (a) insoluble, (b) soluble.

For the soluble surfactant system, a representative snapshot of the positions of surfactant molecules is in Fig. 11. The measured isotherm is shown in Fig. 2, and has the same qualitative features as in the insoluble case. In this figure, the surfactant surface coverage is deter- 
mined by considering the component density profiles as a function of distance normal to the interface, Fig. 4. The upper curve is the solvent profile, while the lower one refers to the tail groups, and the surface coverage $\Gamma$ is taken to be the total area under the bump to the left of $z_{1}$.

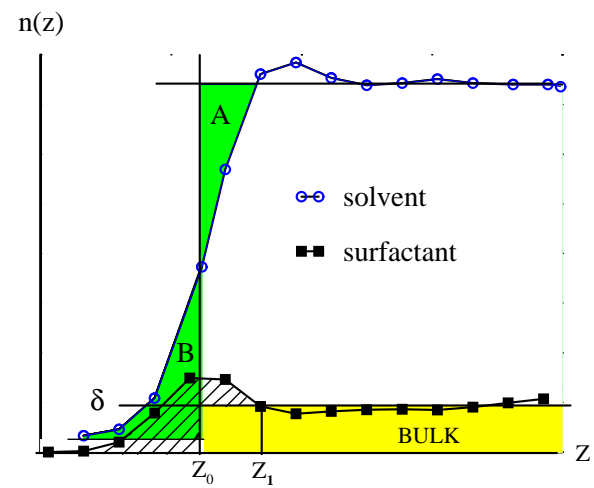

FIG. 4. Interface profile obtained in the simulation, and the Gibbs construction.

To obtain the surfactant adsorption isotherm in a thermodynamically unambiguous manner, we use the density profiles to extract Gibbs" "surface excess concentration" $\Gamma_{e}$ [1]. We first locate a Gibbs dividing surface, $z_{0}$, which serves as a convenient definition of the solvent interface. Referring to Fig. 4 , the location of this surface is defined by the condition that the areas of the shaded regions $A$ and $B$ are equal, thereby requiring the surface excess of solvent to vanish. The bulk surfactant concentration is defined by extrapolating the average bulk density $\delta$ in the bulk up to the Gibbs dividing surfaces,

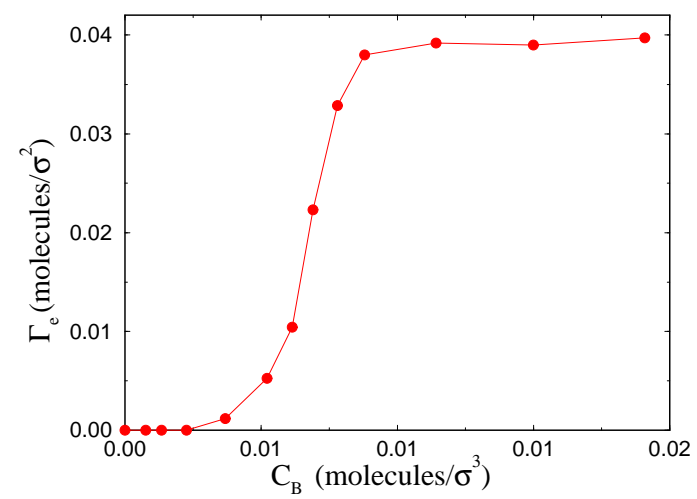

FIG. 5. Surface concentration vs. Bulk concentration.

and the surface excess concentration of surfactant $\Gamma_{e}$ is then the remainder, - the area of the hatched region in the figure. (We assume that there is no surfactant in the vapor phase.) Repeating this procedure at each value of bulk concentration, we obtain the variation of surface excess coverage with total concentration shown in Fig. 5: a monotonic increase, with a sigmoidal shape. Note that the steeply rising part of the curve occurs precisely in the plateau region of the isotherm, which we will argue corresponds to the G/LE phase transition. In the thermodynamic limit one would expect a vertical line there, as surfactant is expelled from solution to form larger liquidlike clusters on the interface, but we observe the smoothing and finite slope characteristic of phase transitions in finite-sized systems.

Returning to the soluble isotherm, note that the critical densities of the gas and liquid phases for the G/LE transition (the end points of the plateau) occur near the same values of $\Gamma$ found for the insoluble case, presumably because this value corresponds to the onset of clustering, and is determined largely by the attraction between surfactant molecules, which was not changed. The pair distribution function for systems at the start, middle and end of the surface tension plateau are shown in Fig. 2p b, and generally resemble the insoluble case. The characterization of these phases is facilitated by examining snapshots of the molecular configuration, and three typical examples corresponding to the three surface coverage values where $g(r)$ was plotted are shown in Fig. 6.

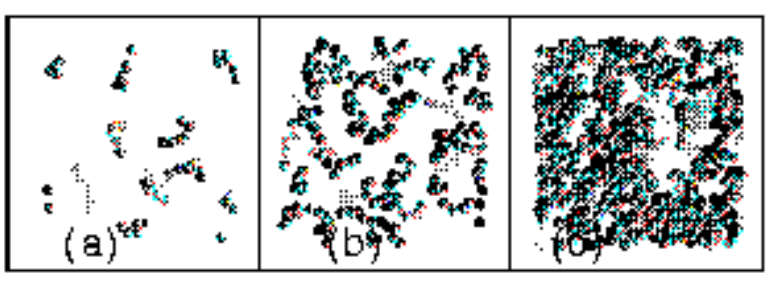

FIG. 6. Snapshots of soluble surfactant molecules at an interface: (a) gas,(b) gas-liquid and (c) liquid expanded phases.

At low surface concentration, Fig. 6a at $\Gamma=0.014$, one sees a gaseous state with isolated molecules and a weak tendency to bind into pairs. At higher coverage $\Gamma=0.062$ in the middle of the plateau, Fig. 6 b, we see a spanning cluster of surfactant molecules, accompanied by isolated molecules. Finally at the end of the plateau, $\Gamma=0.097$ in Fig. 6c, there is a single surfactant monolayer.

Further evidence for phase transitional behavior in the plateau region is obtained by studying the degree of ordering of the surfactant tails. A simple quantitative measurement is the histogram of the tilt angle between the surfactant tail and the normal to the interface, shown in Fig. 7. The orientation of a molecule is defined as the direction of the eigenvector of its moment of inertia tensor with the smallest eigenvalue [5]. We see that as the surface coverage increases across the plateau, molecules are increasingly likely to be oriented normal to the interface. An even more dramatic variation is seen in the histogram of end-to-end lengths, shown in the inset to Fig. If - as coverage increases, the molecules are increasingly extended as they pack together. Note that because the 
molecular interaction corresponds to freely-jointed chains with no bond-bending forces, the surfactant molecules need not form straight rods, and we do not observe complete correlation of the tails seen in MD simulations of condensed phases in Langmuir monolayers [5]. Furthermore, since the tails are now partially soluble they are more likely to have solvent around them, which tends to decorrelate their orientation.

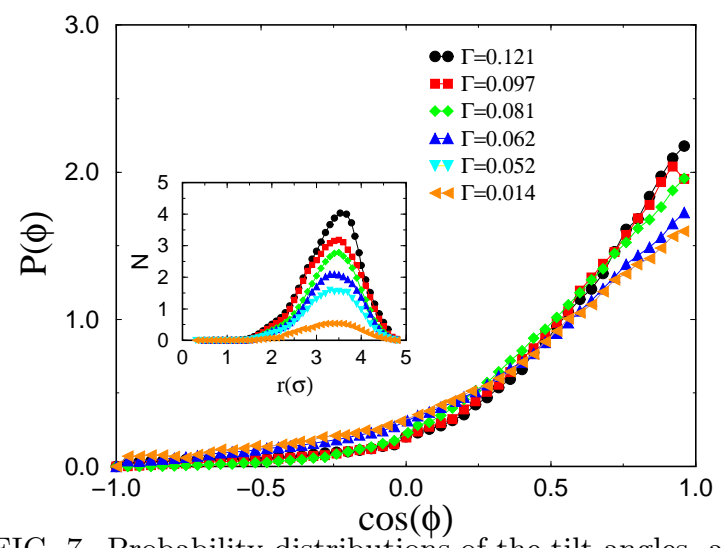

FIG. 7. Probability distributions of the tilt angles, at various values of surface concentration. The inset shows the corresponding histogram of the length of the molecules' end-to-end vectors.

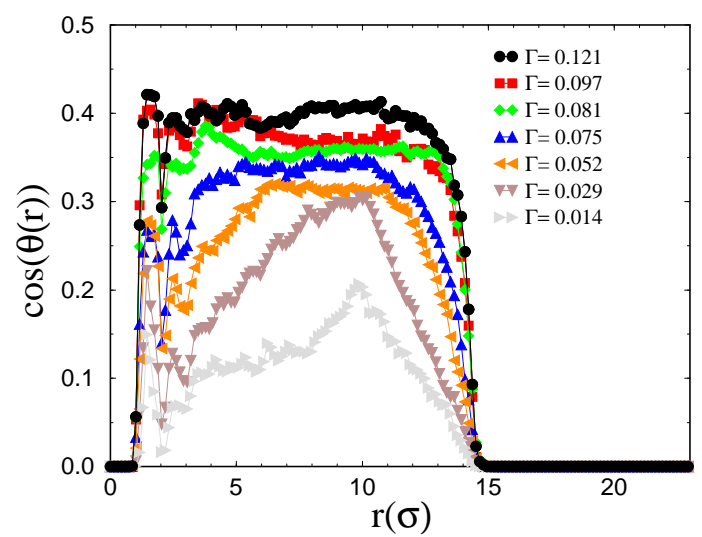

FIG. 8. Orientational correlation function at various coverages.

Lastly, we can go further in this vein and define a pair angular correlation function: the probability distribution for the mean relative angle between two molecular tails as a function of the head group separation $r$. Results are shown in Fig. 8: at low coverage, there is little correlation between the tails, but the likelihood of alignment increases with $\Gamma$, as the molecules pack together and the tail are attracted to each other.

In summary, we have presented MD simulations of soluble amphiphilic systems, containing simple chain molecules in a monatomic bulk solvent, which assem- ble from solution into a surfactant layer at the liquidvapor interface. As a function of surface coverage, these systems exhibit a G/LE phase transition which can be characterized by plateaus in the surface tension isotherm, visible changes in the molecular configurations, and corresponding transitions in various correlation functions. The behavior of insoluble and soluble systems is found to be very similar, suggesting that at least this surface ordering phenomenon is not highly sensitive to the dynamics in the bulk solution, but rather an effect of surface packing.

We thank H. A. Makse for discussions, S. McNamara for his collaboration in the early stages of this work, NASA and NSF for financial support, and NPACI for computer resources.

[1] A. W. Adamson and A. P. Gast, Physical Chemistry of Surfaces, 6th ed. (Wiley, New York, 1997).

[2] C. M. Knobler and R. C. Desai, Annu. Rev. Phys. Chem. 43 207, (1992).

[3] V. M. Kaganer, H. Möhwald and P. Dutta, Rev. Mod. Phys. 71, 779 (1999).

[4] J. P. Bareman and M. L. Klein, J. Phys. Chem. 94, 5202 (1990), and earlier references therein.

[5] S. Karaborni and S. Toxvaerd, J. Chem Phys. 96, 5505 (1992);

[6] M. E. Schmidt, S. Shin and S. A. Rice, J. Chem Phys. 104, 2114 (1996), and earlier references therein.

[7] S. Hénon and J. Meunier, J. Chem. Phys. 98, 9148 (1993); D. Volhardt and V. Meltzer, J. Phys. Chem. 101, 3770 (1997); M. Pollard, R. Pan, C. Steiner and C. Maldarelli, Langmuir 14, 7222 (1998).

[8] J. Böcker, M. Schlenkrich, P. Bopp and J. Brickmann, J. Phys. Chem. 96, 9915 (1992) and M. Tarek, D. J. Tobias and M. L. Klein, J. Phys. Chem. 99, 1393 (1995); V. P. Sokhan and D. J. Tildesley, Faraday Disc. 104, 193 (1996); H. Kuhn and H. Rehage, J. Phys. Chem. B 103, 8493 (1999).

[9] B. Smit, Phys. Rev. A 37, 3431 (1988), and K. Esselink, P.A.J. Hilbers, N.M. van Os, B. Smit and S. Karaborni, Colloids and Surfaces 91, 155 (1994).

[10] M. S. Tomassone, A. Couzis, C. Maldarelli, J. R. Banavar and J. Koplik, to be submitted to J. Chem. Phys.

[11] M.P. Allen and D.J. Tildesley, Computer Simulation of Liquids, (Oxford, Clarendon Press, 1987).

[12] R. B. Bird, C. F. Curtiss, R. C. Armstrong and O. Hassager, Dynamics of Polymeric Liquids, 2nd ed., vol. 2 Kinetic Theory (Wiley, New York, 1987),

[13] J. S. Rowlinson and B. Widom, Molecular Theory of Capillarity (Clarendon, Oxford, (1982).

[14] J. P. Hansen and I. R. Mc. Donald, Theory of Simple Liquids, (Academic Press, New York, 1976). 